\title{
RINOPLASTIA REPARADORA COM ENXERTO DE CARTILAGEM COSTAL EM CASO DE NECROSE SEPTAL EM PACIENTE COM GRANILOMATOSE COM POLIANGEITE
}

\author{
REPAIRING RHINOPLASTY WITH COSTAL CARTILAGE GRAFT IN CASE OF SEPTAL \\ NECROSIS IN A PATIENT WITH GRANILOMATOSIS WITH POLYANGIITIS
}

\begin{abstract}
David Almeida de Souza ${ }^{1 *}$; Maria Elena Mandarim de Lacerda ${ }^{\text {; }}$ Julyane Alves de Assis dos Santos Pereira '; Lara Gomes Botelho 1; Leonardo Ferraz Marques D'Oliveira 1; Leonardo Araujo Guimarães 2. ${ }^{1}$ Acadêmicos de medicina da Faculdade de Medicina de Campos

${ }^{2}$ Médico do Serviço de Cirurgia Plástica do Hospital Universitário Pedro Ernesto, da Universidade Estadual do Rio de Janeiro. Membro Especialista e Titular da Sociedade Brasileira de Cirurgia Plástica.
\end{abstract}

Instituições no qual o trabalho foi realizado: Faculdade de Medicina de Campos. Av. Alberto Torres, 217 - Centro, Campos dos Goytacazes - RJ, 28035-581. Hospital Universitário Pedro Ernesto. Av. Boulevard 28 de Setembro, 77 - Vila Isabel, Rio de Janeiro - RJ, 20551-030

\section{RESUMO}

Descrita em 1937, por Friedrich Wegener, a Granulomatose com Poliangeíte (GPA) anteriormente conhecida como Granulomatose de Wergener - é uma condição autoimune rara. Trata-se de uma vasculite que acomete vasos de pequeno a médio calibre e inflamação granulomatosa necrotizante. Vasos de grande calibre raramente são afetados. Acomete regiões de cabeça e pescoço com regular frequência. Possui predileção pelo trato respiratório alto, pulmões e rins, no entanto pode acometer qualquer órgão. As regiões de orelha, nariz e garganta são as mais comuns em manifestações primárias da doença. Objetivo: Relatar a abordagem cirúrgica reparadora em quadro de deformidade nasal, em paciente com diagnóstico de GPA há 25 anos. Descrições: Paciente do sexo feminino, 41 anos de idade com diagnóstico de GPA há 25 anos. Apresenta quadro de deformidade nasal há aproximadamente 19 anos. Apresenta desabamento do arcabouço cartilaginoso desencadeada por necrose de septo nasal. Não foram relatadas queixas funcionais. Programada rinoplastia aberta com confecção de neosepto em $L$ a partir de enxerto de

\section{ABSTRACT}

Described in 1937 by Friedrich Wegener, Granulomatosis with Polyangeitis (GPA) - formerly known as Wergener's granulomatosis - is a rare autoimmune condition. It is a vasculitis that affects small to medium vessels and necrotizing granulomatous inflammation. Vessels of great caliber are rarely affected. It attacks head and neck regions regularly. It has predilection for the upper respiratory tract, lungs and kidneys, however it can affect any organ. The ear, nose and throat regions are the most common in primary manifestations of the disease. Objective: To report the surgical approach to repairing nasal deformity in a patient diagnosed with GPA 25 years ago. Descriptions: Female patient, 41 years of age with diagnosis of GPA 25 years ago. She presented a picture of nasal deformity about 19 years ago. It presents collapse of the cartilaginous framework triggered by nasal septal necrosis. No functional complaints were reported. Scheduled open rhinoplasty with $L$ neosepto preparation from costal cartilage graft. Currently, the patient has nasal tip support, complete reversal of the nose frame in the saddle, without functional complaints.

* Autor correspondente: David Almeida de Souza. Av. Alberto Torres, 217 - Centro, Campos dos Goytacazes - RJ, 28035-581. Telefone: (22) 2101-2906. E-mail: davidsouzamed@gmail.com. 
cartilagem costal. Atualmente a paciente apresenta sustentação da ponta nasal, reversão completa do quadro de nariz em sela, sem queixas funcionais. Conclusão: A GPA é uma doença rara que em acometimentos nasais a perfuração de septo é um evento comum. Raramente, com um longo tempo de doença, pode causar destruição mais extensa da cartilagem nasal, gerando deformidades. Este artigo apresenta um caso raro, de uma paciente com longo período de doença, apresentando deformidade nasal. Foi realizada rinoplastia reparadora com enxerto de cartilagem costal. A cirurgia foi realizada no Serviço de Cirurgia Plástica do Hospital Universitário Pedro Ernesto.

Descritores: Cirurgia Plástica, Granulomatose com Poliangiite, Poliangiite Microscópica, Rinoplastia.

\section{INTRODUÇÃO}

Descrita em 1937, por Friedrich Wegener, a Granulomatose com Poliangeíte (GPA) anteriormente conhecida como Granulomatose de Wergener - é uma condição autoimune rara, , com uma prevalência de 3 casos a cada 100.000 habitantes nos Estados Unidos. É classificada no grupo das vasculites associadas ao ANCA- anticorpo antineutrófilo citoplasmático.

Fisiopatologicamente, trata-se de uma vasculite que acomete vasos de pequeno a médio calibre e inflamação granulomatosa necrotizante Vasos de grande calibre raramente são afetados. Acomete regiões de cabeça e pescoço com regular frequência. Possui predileção pelo trato respiratório alto, pulmões e rins, no entanto pode acometer qualquer órgão.

As regiões de orelha, nariz e garganta são as mais comuns em manifestações primárias da doença. Quando limitada ao trato respiratório superior, o diagnóstico diferencial inclui condições como infecções (espiroquetas, mycobactérias, bactérias, fungos) e outras condições inflamatórias como sarcoidose, Churg-Strauss, e destruição do septo nasal por uso abusivo de cocaína.

Um estudo realizado pelo Grupo Europeu de Estudo de Vasculites (EUVAS) sugeriu uma classificação para os pacientes de acordo com os níveis de gravidade da doença, variando de doença localizada, sistêmica, generalizada, grave, e forma
Conclusion: GPA is a rare disease that in nasal attacks, septum perforation is a common event. Rarely, with a long time of illness, it can cause more extensive destruction of the nasal cartilage, generating deformities. This article presents a rare case of a patient with a long period of disease, presenting nasal deformity. A repair rhinoplasty with costal cartilage graft was performed. The surgery was performed at the Plastic Surgery Service of University Hospital Pedro Ernesto.

Keywords: Granulomatosis with Polyangiitis, Microscopic Polyangiitis, Rhinoplasty, Plastic,Surgery.

refratária, dependendo do tipo, localização e gravidade das manifestações clínicas e propos diferentes protocolos específicos para cada um dos níveis.

O objetivo do trabalho foi relatar a abordagem cirúrgica reparadora em quadro de deformidade nasal, ocasionada pelo desabamento de arcabouço cartilaginoso nasal, em paciente com diagnóstico de GPA há 25 anos.

\section{RELATO DE CASO}

Paciente do sexo feminino, 41 anos de idade com diagnóstico de GPA há 25 anos. Apresenta quadro de deformidade nasal há aproximadamente 19 anos. Refere ainda infecções de repetição do trato respiratório superior. Apresenta desabamento do arcabouço cartilaginoso desencadeada por necrose de septo nasal. Não foram relatadas queixas funcionais.

Ao exame apresenta nariz curto com rebordo ósseo evidente. Desabamento de arcabouço cartilaginoso gerando ponta de nariz sem sustentação (nariz em sela).

Programada rinoplastia aberta com confecção de neosepto em $L$ a partir de enxerto de cartilagem costal. A cirurgia foi realizada no Serviço de Cirurgia Plástica do Hospital Universitário Pedro Ernesto. No ato cirúrgico, esperava-se realizar o reposicionamento das cartilagens triangulares, seguido de reforço da válvula nasal externa com 
enxertos de sobreposição de cartilagem alar. No entanto no ato operatório notou-se completa ausência de cartilagem septal (figura 1) e grande fibrose de triangulares apresentando ausência de mobilidade do forro nasal. As cartilagens alares apresentavam pouca projeção, acompanhando a pouca mobilidade superior das triangulares.

Foi realizada a retirada da cartilagem costal (figura 2A) e confeç̧ão do enxerto em L (figura 2B).

Realizado reposicionamento das cartilagens triangulares, o mais superior possível, no entanto pela magnitude da fibrose, não foi possível alcançar o neosepto. Portanto, lançou-se mão da realização de enxertos de sobreposição, tanto na cartilagem triangular como na alar, conforme ilustração (figura 3). Observado resultado satisfatório no pós-cirúrgico imediato. A paciente seguiu acompanha-mento ambulatorial, sem complicações.

O resultado após um ano foi considerado de sucesso. Paciente apresenta sustentação da ponta nasal, reversão completa do quadro de nariz em sela, sem queixas funcionais (Figura 4A, 4B e 4C).

\section{DISCUSSÃO:}

O acometimento da mucosa respiratória nasal é um evento comum em desordens autoimunes, mais frequentemente observadas na GPA Quando existe acometimento do tecido cartilaginoso, é possível que ocorra perfuração do septo nasal inicialmente, progressivamente podendo acometer toda cartilagem nasal causando deformidades estruturais, como relatado no caso índice.

Acometimento nasal também já foi descrito, porém menos frequentemente em lúpus eritematoso sistêmico, crioglobulinemias, doença de crohn e artrite reumatoide e outras afecções autoimunes.

Os sintomas mais comumente associados ao acometimento nasal são crostas de mucosa nasal, epistaxe, rinorreia. De forma menos frequente, obstrução nasal, disosmia, rinite, sinusite e cefaleia. A ocorrência de epífora e deformidade de cartilagem nasal - nariz em sela - são eventos com menor incidência e apresentam um maior desafio na abordagem clínica e cirúrgica em caso de reparação.

Nos casos onde temos perfuração de septo, o tipo mais comum de cirurgia realizada mundialmente consiste no retalho de mucosa nasal, seguida enxerto de cartilagem. A escolha do tecido para confecção dos enxertos varia, podendo ser utilizados tecidos como cartilagem, fáscia muscular,

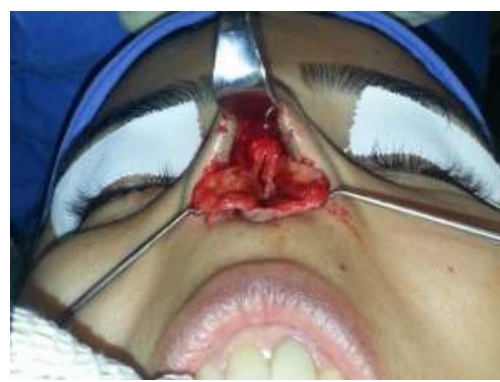

Figura 1- Ausência de cartilagem septal evidenciada durante ato cirúrgico.

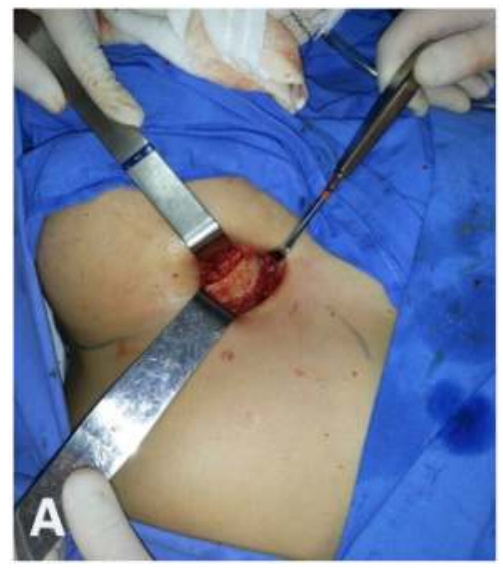

Figura 2

(A) Retirada de cartilagem costal para confecção do Enxerto.

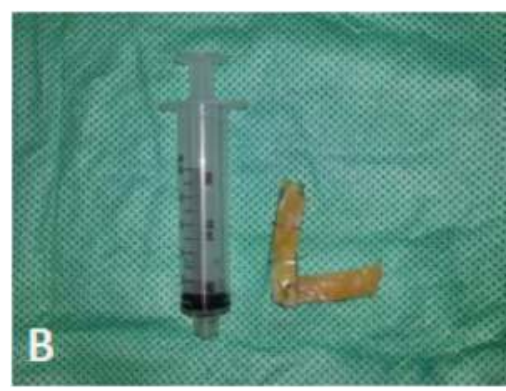
(B) Enxerto em L, para construção de neosepto.

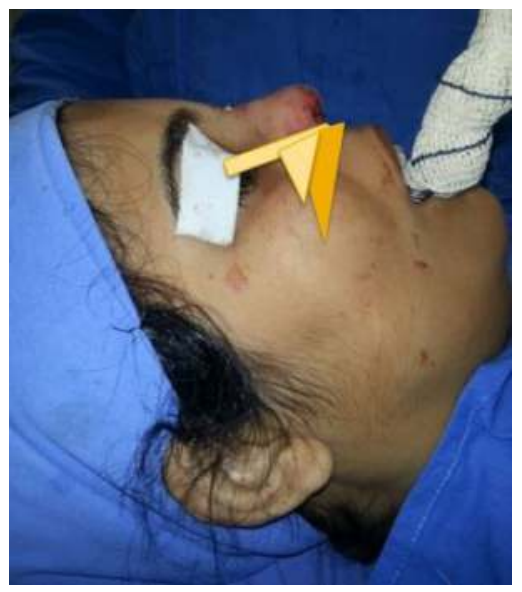

Figura 3

Apresentação esquemática de enxertos de sobreposição de cartilagens triangular e alar.

pele e tecido ósseo. Entre estes, a cartilagem é o tecido mais utilizado na realização deste procedimento6.

A indicação de cirurgia para correção de septo nasal esta relacionada ao tamanho da lesão, não sendo indicada em pacientes como lesões 
menores que $5 \mathrm{~mm}$, a não ser que haja sintomatologia associada. As principais contraindicações descritas são: Desvio de septo, rinite atrófica, tabagismo, epistaxe, abuso de drogas, perfuração assintomática e idade avançada onde os benefícios não superariam os riscos envolvidos na cirurgia6.

O prognóstico do paciente pós-cirurgia depende de fatores como dimensão da perfuração, habilidade do cirurgião, doenças sistêmicas, inflamação, sangramento, ressecamento da mucosa e atributos relativos ao tabagismo.

Nos pacientes com necrose progressiva do septo e destruição do mesmo, temos que ter em mãos técnicas de reconstrução nasal, que abordem tanto o arcabouço cartilaginoso quanto a mobilização do forro nasal.

A cartilagem costal é a mais indicada nessas situações pela sua abundância e forma. Sendo empregada na construção de um novo septo em formato de L, além de poder gerar enxertos de sobreposição que podem ser moldados de acordo com a necessidade.

\section{CONCLUSÃO:}

As deformidades do nariz podem gerar problemas fisiológicos, por prejudicar a funcionalidade do trato respiratório, assim como sociais pela apresentação estética desses pacientes.

O tratamento precoce da patologia de base deve ser feito. Quanto mais cedo a intervenção das complicações nasais, sejam perfurações ou necroses completas, maiores as chances do cirurgião obter êxito.
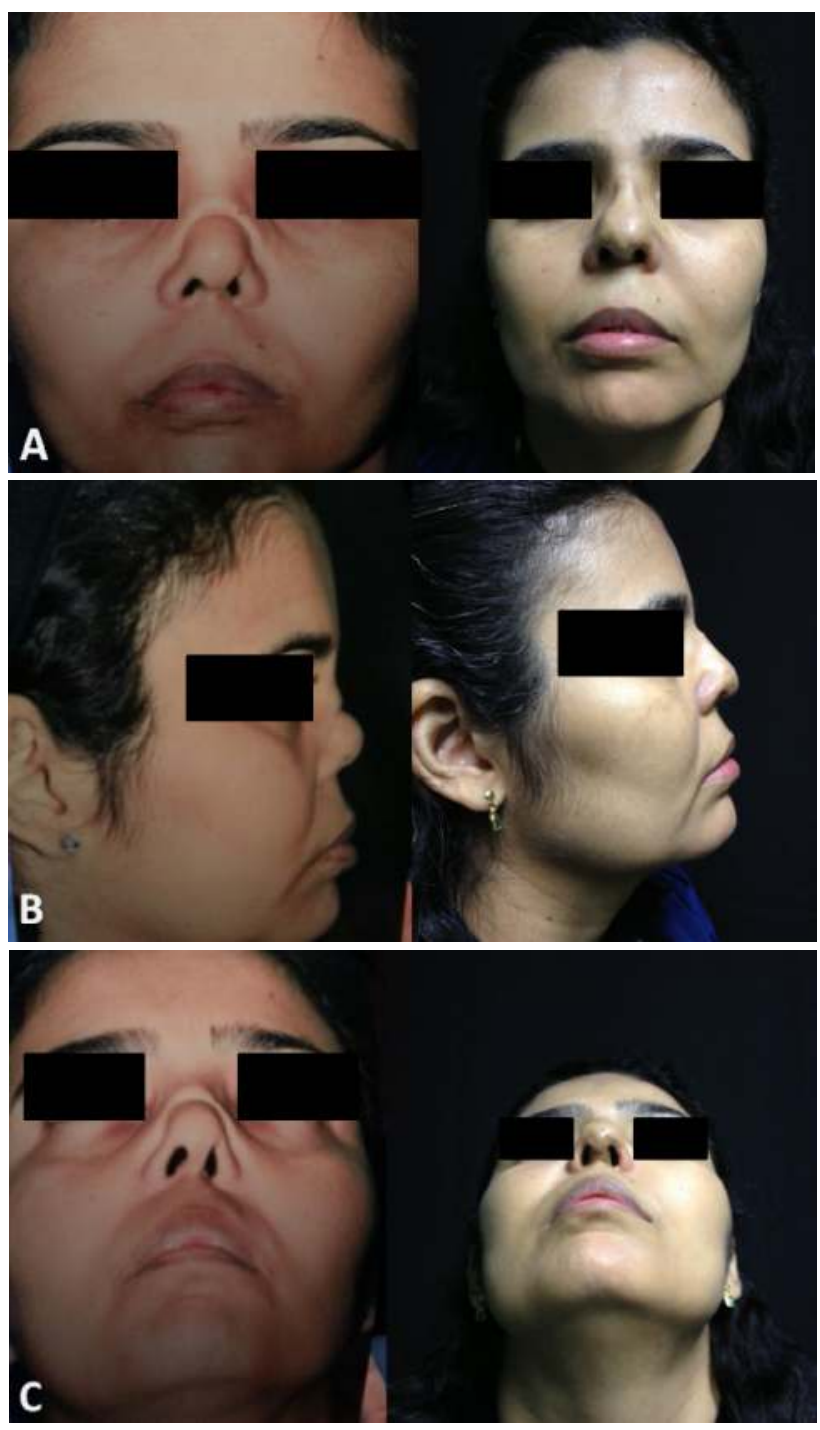

Figura 4 - Resultado comparativo após 1 ano (A) Visão anterior. (B) Visão lateral. (C) Visão inferior.

\section{REFERÊNCIAS:}

1. Guntupalli L, Patel K, Faraji F, Brunworth JD. Autoimmune-related nasal septum perforation: A case report and systematic review. Allergy Rhinol (Providence). 2017;8(1):40-4.

2. Kuhn D, Hospowsky C, Both M, Hey M, Laudien M. Manifestation of granulomatosis with polyangiitis in head and neck. Clin Exp Rheumatol. 2018;36 Suppl 111(2):78-84.

3. Trimarchi M, Sinico RA, Teggi R, Bussi M, Specks U, Meroni PL. Otorhinolaryngological manifestations in granulomatosis with polyangiitis (Wegener's). Autoimmun Rev. 2013;12(4):501-5.

4. Felicetti M, Cazzador D, Padoan R, Pendolino AL, Faccioli C, Nardello E, et al. Ear, nose and throat involvement in granulomatosis with polyangiitis: how it presents and how it determines disease severity and long-term outcomes. Clin Rheumatol. 2018;37(4):1075-83.

5. Sehgal R, Resnick JM, Al-Hilli A, Mehta N, Conway T, Stratman EJ. Nasal septal and mucosal disease associated with pyoderma gangrenosum in a cocaine user. JAAD Case Rep. 2017;3(4):284-7.

6. PASSALI, Desiderio et al. Surgical treatment of nasal septal perforations: SIR (Italian Society of Rhinology) experts opinion . Espanha: Elsevier Espanha, 2017. 1-6 p. v. 764. 